\title{
The Gordian distance of handlebody-knots and Alexander biquandle colorings
}

\author{
By Tomo Murao
}

(Received Feb. 28, 2017)

\begin{abstract}
We give lower bounds for the Gordian distance and the unknotting number of handlebody-knots by using Alexander biquandle colorings. We construct handlebody-knots with Gordian distance $n$ and unknotting number $n$ for any positive integer $n$.
\end{abstract}

\section{Introduction.}

The Gordian distance of two classical knots is the minimal number of crossing changes needed to be deformed each other. In particular, we call the Gordian distance of a classical knot and the trivial one the unknotting number of the classical knot. Clark, Elhamdadi, Saito and Yeatman [2] gave a lower bound for the Nakanishi index [16], which induced a lower bound for the unknotting number of classical knots. This is a generalization of the Przytycki's result [17]. In this paper, we give lower bounds for the Gordian distance and the unknotting number of handlebody-knots, which is a generalization of a classical knot with respect to a genus.

Ishii [4] introduced an enhanced constituent link of a spatial trivalent graph, and Ishii and Iwakiri [6] introduced an $A$-flow of a spatial graph, where $A$ is an abelian group, to define colorings and invariants of handlebody-knots. Iwakiri [12] gave a lower bound for the unknotting number of handlebody-knots by using Alexander quandle colorings of its $\mathbb{Z}_{2}$ or $\mathbb{Z}_{3}$-flowed diagram. Ishii, Iwakiri, Jang and Oshiro [7] introduced a $G$-family of quandles, which is an extension of the above structures. Recently, Ishii and Nelson [11] introduced a $G$-family of biquandles, which is a biquandle version of a $G$-family of quandles.

In this paper, we extend the result in $[\mathbf{1 2}]$ in three directions. First, we extend from $\mathbb{Z}_{2}, \mathbb{Z}_{3}$-flows to any $\mathbb{Z}_{m}$-flow. Second, we extend from quandles to biquandles. Finally, we extend from unknotting numbers to Gordian distances. Thus we can determine the Gordian distance and the unknotting number of handlebody-knots more efficiently. We construct handlebody-knots with Gordian distance $n$ and unknotting number $n$ for any $n \in \mathbb{Z}_{>0}$ and note that one of them can not be obtained by using Alexander quandle colorings introduced in $[\mathbf{1 2}]$.

This paper is organized into seven sections. In Section 2, we recall the definition of a handlebody-knot and introduce the Gordian distance and the unknotting number of handlebody-knots. In Section 3, we recall the definition of a (bi)quandle and a $G$-family

2010 Mathematics Subject Classification. Primary 57M25; Secondary 57M15, 57M27.

Key Words and Phrases. handlebody-knot, biquandle, Gordian distance, unknotting number. 
of (bi)quandles. In Section 4, we introduce a coloring of a diagram of a handlebodyknot by using a $G$-family of biquandles. In Section 5, we show that there are linear relationships for Alexander biquandle colorings of a diagram of a handlebody-knot. In Section 6, we give lower bounds for the Gordian distance and the unknotting number of handlebody-knots by using $\mathbb{Z}_{m}$-family of Alexander biquandles colorings. In section 7 , we construct handlebody-knots with Gordian distance $n$ and unknotting number $n$ for any $n \in \mathbb{Z}_{>0}$. Moreover, we note that one of them can not be obtained by using Alexander quandle colorings with $\mathbb{Z}_{2}, \mathbb{Z}_{3}$-flows introduced in [12].

\section{The Gordian distance of handlebody-knots.}

A handlebody-link, which is introduced in [4], is the disjoint union of handlebodies embedded in the 3 -sphere $S^{3}$. A handlebody-knot is a handlebody-link with one component. In this paper, we assume that every component of a handlebody-link is of genus at least 1. An $S^{1}$-orientation of a handlebody-link is an orientation of all genus 1 components of the handlebody-link, where an orientation of a solid torus is an orientation of its core $S^{1}$. Two $S^{1}$-oriented handlebody-links are equivalent if there exists an orientation-preserving self-homeomorphism of $S^{3}$ sending one to the other preserving the $S^{1}$-orientation.

A spatial trivalent graph is a graph whose vertices are valency 3 embedded in $S^{3}$. In this paper, a trivalent graph may have a circle component, which has no vertices. A $Y$-orientation of a spatial trivalent graph is a direction of all edges of the graph satisfying that every vertex of the graph is both the initial vertex of a directed edge and the terminal vertex of a directed edge (Figure 1). A vertex of a Y-oriented spatial trivalent graph can be allocated a sign; the vertex is said to be positive or negative, or to have sign +1 or -1 . The standard convention is shown in Figure 1. For a Y-oriented spatial trivalent graph $K$ and an $S^{1}$-oriented handlebody-link $H$, we say that $K$ represents $H$ if $H$ is a regular neighborhood of $K$ and the $S^{1}$-orientation of $H$ agrees with the Y-orientation. Then any $S^{1}$-oriented handlebody-link can be represented by some Y-oriented spatial trivalent graph. We define a diagram of an $S^{1}$-oriented handlebody-link by a diagram of a Y-oriented spatial trivalent graph representing the handlebody-link. An $S^{1}$-oriented handlebody-link is trivial if it has a diagram with no crossings. Then the following theorem holds.
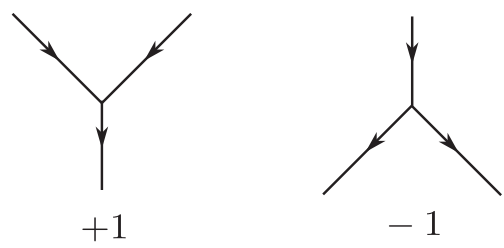

Figure 1. Y-orientations and signs.

Theorem $2.1([\mathbf{5}])$. For a diagram $D_{i}$ of an $S^{1}$-oriented handlebody-link $H_{i}(i=$ $1,2), H_{1}$ and $H_{2}$ are equivalent if and only if $D_{1}$ and $D_{2}$ are related by a finite sequence 

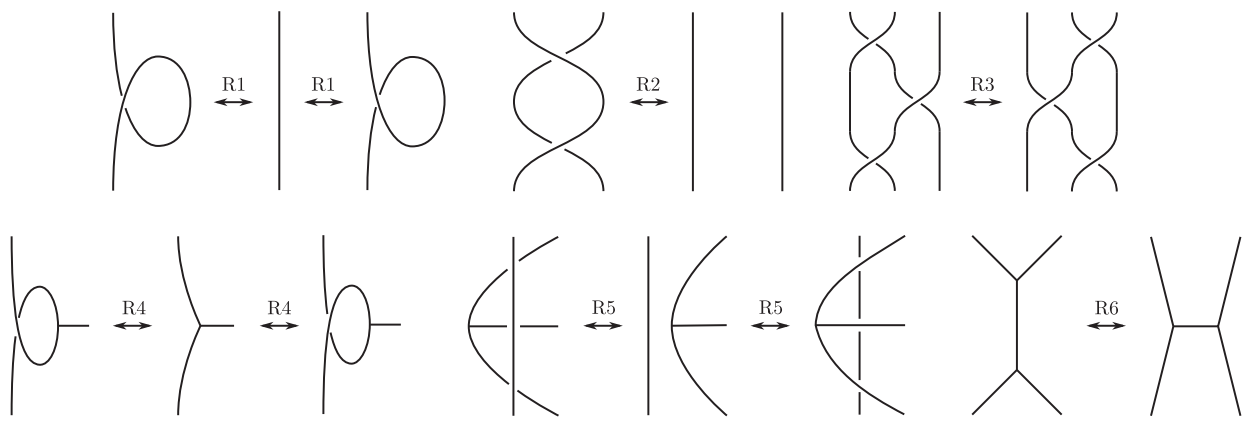

Figure 2. The Reidemeister moves for handlebody-links.

of R1-R6 moves depicted in Figure 2 preserving $Y$-orientations.

In this paper, for a diagram $D$ of an $S^{1}$-oriented handlebody-link, we denote by $\mathcal{A}(D)$ and $\mathcal{S} \mathcal{A}(D)$ the set of all arcs of $D$ and the one of all semi-arcs of $D$ respectively, where a semi-arc is a piece of a curve each of whose endpoints is a crossing or a vertex. An orientation of a (semi-)arc of $D$ is also represented by the normal orientation obtained by rotating the usual orientation counterclockwise by $\pi / 2$ on the diagram. For any $m \in \mathbb{Z}_{\geq 0}$, we put $\mathbb{Z}_{m}:=\mathbb{Z} / m \mathbb{Z}$.

A crossing change of an $S^{1}$-oriented handlebody-link $H$ is that of a spatial trivalent graph representing $H$. This deformation can be realized by switching two handles depicted in Figure 3. It is easy to see that any two $S^{1}$-oriented handlebody-knots of the same genus can be related by a finite sequence of crossing changes. For any two $S^{1}$-oriented handlebody-knots $H_{1}$ and $H_{2}$ of the same genus, we define their Gordian distance $d\left(H_{1}, H_{2}\right)$ by the minimal number of crossing changes needed to be deformed each other. In particular, for any $S^{1}$-oriented handlebody-knot $H$ and the $S^{1}$-oriented trivial handlebody-knot $O$ of the same genus, we define $u(H):=d(H, O)$, which is called the unknotting number of $H$.

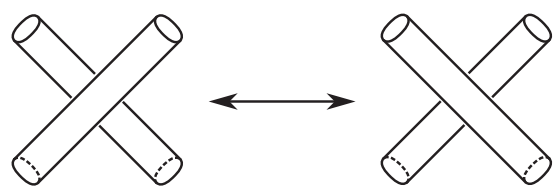

Figure 3. A crossing change of an $S^{1}$-oriented handlebody-link.

\section{A biquandle and a $G$-family of biquandles.}

We recall the definitions of a quandle and a biquandle.

Definition $3.1([\mathbf{1 3}],[\mathbf{1 4}])$. A quandle is a non-empty set $X$ with a binary operation $*: X \times X \rightarrow X$ satisfying the following axioms.

- For any $x \in X, x * x=x$. 
- For any $x \in X$, the map $S_{x}: X \rightarrow X$ defined by $S_{x}(y)=y * x$ is a bijection.

- For any $x, y, z \in X,(x * y) * z=(x * z) *(y * z)$.

Definition $3.2([3])$. A biquandle is a non-empty set $X$ with binary operations $\bar{*}, \underline{*}: X \times X \rightarrow X$ satisfying the following axioms.

- For any $x \in X, x \underline{*} x=x \bar{\star} x$.

- For any $x \in X$, the map $\underline{S}_{x}: X \rightarrow X$ defined by $\underline{S}_{x}(y)=y \underline{*} x$ is a bijection.

For any $x \in X$, the map $\bar{S}_{x}: X \rightarrow X$ defined by $\bar{S}_{x}(y)=y \bar{\star} x$ is a bijection.

The map $S: X \times X \rightarrow X \times X$ defined by $S(x, y)=(y \mp x, x \underline{*} y)$ is a bijection.

- For any $x, y, z \in X$,

$$
\begin{aligned}
& (x \underline{*} y) \text { * }(z \underline{*} y)=(x \underline{*} z) *(y \bar{*} z), \\
& (x \text { ㄴy } y) \bar{*}(z \text { } y)=(x \bar{*} z) \text { }(y \bar{*} z) \text {, } \\
& (x \bar{*} y) \bar{*}(z \bar{*} y)=(x \bar{*} z) \bar{*}(y \underline{*} z) \text {. }
\end{aligned}
$$

We define $\underline{*}^{n} x:=\underline{S}_{x}^{n}$ and $\bar{*}^{n} x:=\bar{S}_{x}^{n}$ for any $n \in \mathbb{Z}$. We note that $(X, *)$ is a quandle if and only if $(X, *, \bar{*})$ is a biquandle with $x \bar{*} y=x$. For any $m \in \mathbb{Z}_{\geq 0}$, a $\mathbb{Z}_{m}\left[s^{ \pm 1}, t^{ \pm 1}\right]$ module $X$ is a biquandle with $a * b=t a+(s-t) b$ and $a \bar{*} b=s a$, which we call an Alexander biquandle. When $s=1$, an Alexander biquandle coincides with an Alexander quandle.

Definition $3.3([\mathbf{8}])$. Let $X$ be a biquandle. We define two families of binary operations $\underline{*}^{[n]}, \bar{*}^{[n]}: X \times X \rightarrow X(n \in \mathbb{Z})$ by the equalities

$$
\begin{aligned}
& a \underline{*}^{[0]} b=a, a \underline{*}^{[1]} b=a \underline{*}^{[1} b, a \underline{*}^{[i+j]} b=\left(a \underline{*}^{[i]} b\right) \underline{*}^{[j]}\left(b \underline{*}^{[i]} b\right), \\
& a \bar{*}^{[0]} b=a, a \bar{*}^{[1]} b=a \bar{*} b, a \bar{*}^{[i+j]} b=\left(a \bar{*}^{[i]} b\right) \bar{*}^{[j]}\left(b \bar{*}^{[i]} b\right)
\end{aligned}
$$

for any $i, j \in \mathbb{Z}$.

Since $a=a \underline{*}^{[0]} b=\left(a \underline{*}^{[-1]} b\right) \underline{*}^{[1]}\left(b \underline{*}^{[-1]} b\right)=\left(a \underline{*}^{[-1]} b\right) \underline{*}\left(b \underline{*}^{[-1]} b\right)$, we have $a \underline{*}^{[-1]} b=a \underline{*}^{-1}\left(b \underline{*}^{[-1]} b\right)$ and $\left(b \underline{*}^{[-1]} b\right) \underline{*}\left(b \underline{*}^{[-1]} b\right)=b$. Then for an Alexander biquandle $X$, we have $a \underline{*}^{[n]} b=t^{n} a+\left(s^{n}-t^{n}\right) b$ and $a \bar{*}^{[n]} b=s^{n} a$ for any $a, b \in X$.

We define the type of a biquandle $X$ by

$$
\text { type } X=\min \left\{n>0 \mid a \underline{*}^{[n]} b=a=a \bar{\star}^{[n]} b(\forall a, b \in X)\right\} .
$$

Any finite biquandle is of finite type $[\mathbf{1 1}]$.

We also recall the definitions of a $G$-family of quandles and a $G$-family of biquandles.

Definition $3.4([\mathbf{7}])$. Let $G$ be a group with the identity element $e$. A $G$-family of quandles is a non-empty set $X$ with a family of binary operations $*^{g}: X \times X \rightarrow X(g \in G)$ satisfying the following axioms. 
- For any $x \in X$ and $g \in G, x *^{g} x=x$.

- For any $x, y \in X$ and $g, h \in G, x *^{g h} y=\left(x *^{g} y\right) *^{h} y$ and $x *^{e} y=x$.

- For any $x, y, z \in X$ and $g, h \in G,\left(x *^{g} y\right) *^{h} z=\left(x *^{h} z\right) *^{h^{-1} g h}\left(y *^{h} z\right)$.

Definition $3.5([\mathbf{8}],[\mathbf{1 1}])$. Let $G$ be a group with the identity element $e$. A $G$-family of biquandles is a non-empty set $X$ with two families of binary operations $\underline{*}^{g}, \bar{*}^{g}: X \times X \rightarrow X(g \in G)$ satisfying the following axioms.

- For any $x \in X$ and $g \in G$,

$$
x \underline{*}^{g} x=x{\varlimsup^{g}}^{g} .
$$

- For any $x, y \in X$ and $g, h \in G$,

$$
\begin{aligned}
& x \underline{*}^{g h} y=\left(x{\underline{*}^{g}}^{g} y\right){\mathfrak{*}^{h}}^{h}\left(y \underline{*}^{g} y\right), x \underline{*}^{e} y=x, \\
& x \bar{*}^{g h} y=\left(x \bar{*}^{g} y\right) \bar{*}^{h}\left(y \bar{*}^{g} y\right), x \bar{*}^{e} y=x .
\end{aligned}
$$

- For any $x, y, z \in X$ and $g, h \in G$,

$$
\begin{aligned}
& \left(x \underline{*}^{g} y\right){\varkappa^{h}}^{h}\left(z \bar{*}^{g} y\right)=\left(x \underline{*}^{h} z\right){\varkappa^{h}}^{-1} g h\left(y \underline{*}^{h} z\right), \\
& \left(x \bar{*}^{g} y\right) \underline{*}^{h}\left(z \bar{*}^{g} y\right)=\left(x \underline{*}^{h} z\right) \bar{*}^{h^{-1} g h}\left(y \underline{*}^{h} z\right), \\
& \left(x \bar{*}^{g} y\right) \bar{*}^{h}\left(z \bar{*}^{g} y\right)=\left(x \bar{*}^{h} z\right) \bar{*}^{h^{-1} g h}\left(y \underline{*}^{h} z\right) .
\end{aligned}
$$

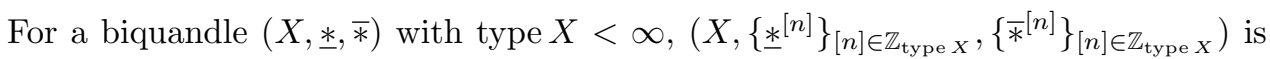

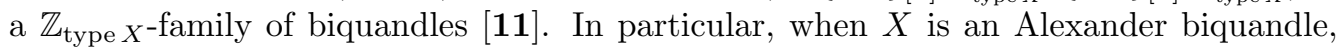

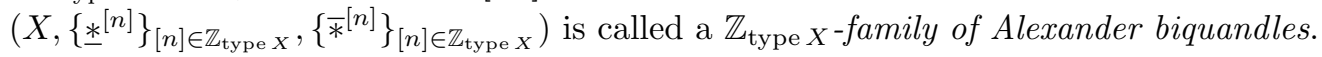

\section{Colorings.}

In this section, we introduce a coloring of a diagram of an $S^{1}$-oriented handlebodylink by a $G$-family of biquandles. Let $G$ be a group and let $D$ be a diagram of an $S^{1}$-oriented handlebody-link $H$. A $G$-flow of $D$ is a map $\phi: \mathcal{A}(D) \rightarrow G$ satisfying
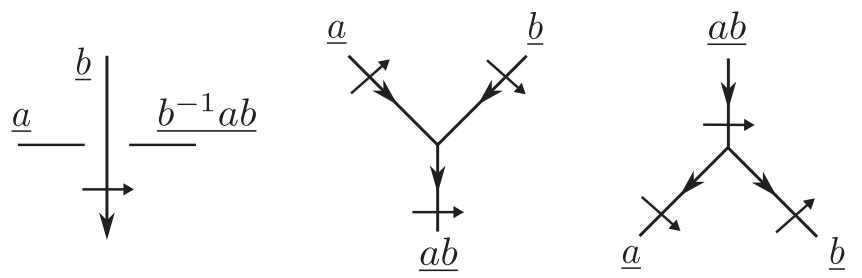

at each crossing and each vertex. In this paper, to avoid confusion, we often represent an element of $G$ with an underline. We denote by $(D, \phi)$, which is called a $G$-flowed diagram of $H$, a diagram $D$ given a $G$-flow $\phi$ and put $\operatorname{Flow}(D ; G):=\{\phi \mid \phi: G$-flow of $D\}$. We can identify a $G$-flow $\phi$ with a homomorphism from the fundamental group $\pi_{1}\left(S^{3}-H\right)$ to $G$. 
Let $G$ be a group and let $D$ be a diagram of an $S^{1}$-oriented handlebody-link $H$. Let $D^{\prime}$ be a diagram obtained by applying one of Reidemeister moves to the diagram $D$ once. For any $G$-flow $\phi$ of $D$, there is an unique $G$-flow $\phi^{\prime}$ of $D^{\prime}$ which coincides with $\phi$ except near the point where the move applied. Therefore the number of $G$-flow of $D$, denoted by \#Flow $(D ; G)$, is an invariant of $H$. We call the $G$-flow $\phi^{\prime}$ the associated $G$-flow of $\phi$ and the $G$-flowed diagram $\left(D^{\prime}, \phi^{\prime}\right)$ the associated $G$-flowed diagram of $(D, \phi)$.

For any $m \in \mathbb{Z}_{\geq 0}$ and $\mathbb{Z}_{m}$-flow $\phi$ of a diagram $D$ of an $S^{1}$-oriented handlebody-link $H$, we define $\operatorname{gcd} \phi:=\operatorname{gcd}\{\phi(a), m \mid a \in \mathcal{A}(D)\} \in \mathbb{Z}_{\geq 0}$, where we regard $\phi(a)$ as an arbitrary element of $\mathbb{Z}$ which represents $\phi(a) \in \mathbb{Z}_{m}$. Then we have the following lemma in the same way as in $[\mathbf{9}]$.

Lemma 4.1. For any $m \in \mathbb{Z}_{\geq 0}$, let $(D, \phi)$ be a $\mathbb{Z}_{m}$-flowed diagram of an $S^{1}$-oriented handlebody-link $H$ and let $\left(D^{\prime}, \phi^{\prime}\right)$ be the associated $\mathbb{Z}_{m}$-flowed diagram of $(D, \phi)$. Then it follows that $\operatorname{gcd} \phi=\operatorname{gcd} \phi^{\prime}$.

Let $G$ be a group, $X$ be a $G$-family of biquandles and let $(D, \phi)$ be a $G$-flowed diagram of an $S^{1}$-oriented handlebody-link $H$. An $X$-coloring of $(D, \phi)$ is a map $C$ : $\mathcal{S} \mathcal{A}(D, \phi) \rightarrow X$ satisfying
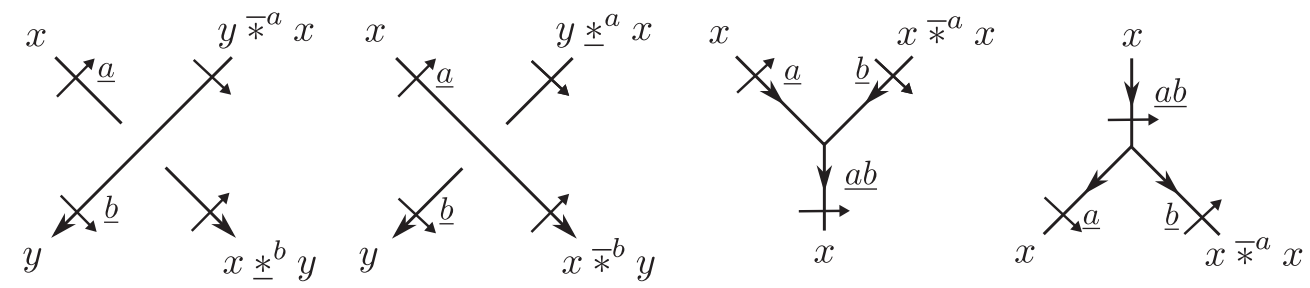

at each crossing and each vertex, where $\mathcal{S} \mathcal{A}(D, \phi)$ is the set of all semi-arcs of $(D, \phi)$. We denote by $\operatorname{Col}_{X}(D, \phi)$ the set of all $X$-colorings of $(D, \phi)$. We note that $\operatorname{Col}_{X}(D, \phi)$ is a vector space over $X$ when $X$ is a $\mathbb{Z}_{m}$-family of Alexander biquandles and a field.

Proposition $4.2([\mathbf{1 1}])$. Let $X$ be a $G$-family of biquandles and let $(D, \phi)$ be a $G$-flowed diagram of an $S^{1}$-oriented handlebody-link $H$. Let $\left(D^{\prime}, \phi^{\prime}\right)$ be the associated $G$ flowed diagram of $(D, \phi)$. For any $X$-coloring $C$ of $(D, \phi)$, there is an unique $X$-coloring $C^{\prime}$ of $\left(D^{\prime}, \phi^{\prime}\right)$ which coincides with $C$ except near the point where the move applied.

We call the $X$-coloring $C^{\prime}$ the associated $X$-coloring of $C$. By this proposition, we have $\# \operatorname{Col}_{X}(D, \phi)=\# \operatorname{Col}_{X}\left(D^{\prime}, \phi^{\prime}\right)$.

Proposition 4.3. Let $G$ be a group and let $X$ be a $G$-family of biquandles. Then the following hold.

1. Let $(D, \phi)$ be a $G$-flowed diagram of an $S^{1}$-oriented handlebody-link. Then it follows that $\# \mathrm{Col}_{X}(D, \phi) \geq \# X$.

2. Let $(O, \psi)$ be a $G$-flowed diagram of an $S^{1}$-oriented m-component trivial handlebody-link. Then it follows that $\# \mathrm{Col}_{X}(O, \psi)=(\# X)^{m}$. 


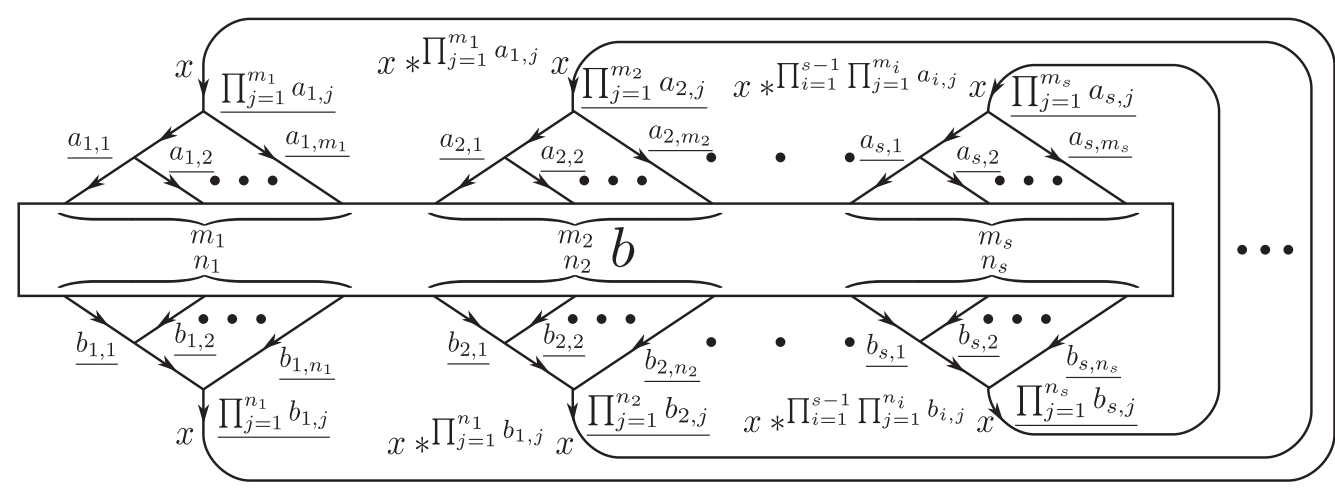

$\left(D^{\prime}, \phi^{\prime}\right)$

Figure 4. A $G$-flowed diagram $\left(D^{\prime}, \phi^{\prime}\right)$ and its $X$-coloring.

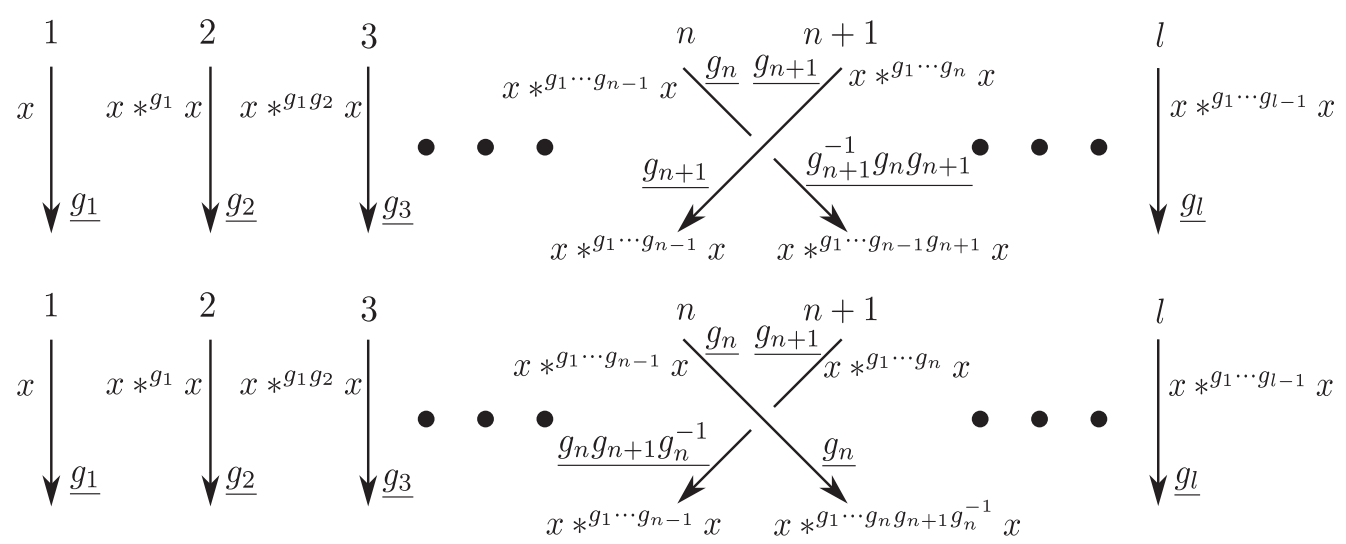

Figure 5. An $X$-coloring of $\left(D^{\prime}, \phi^{\prime}\right)$ in the part of $b$.

Proof. 1. By Theorem 2.1 and [15], we can deform $(D, \phi)$ into the $G$ flowed diagram $\left(D^{\prime}, \phi^{\prime}\right)$ depicted in Figure 4 by a finite sequence of Reidemeister moves preserving Y-orientations, where $b$ is a classical $l$-braid, and $a_{i, 1}, \ldots, a_{i, m_{i}}, b_{i, 1}, \ldots, b_{i, n_{i}} \in G$ for any $i=1, \ldots, s$. We note that $\prod_{j=1}^{m_{i}} a_{i, j}=$ $\prod_{j=1}^{n_{i}} b_{i, j}$ for any $i=1, \ldots, s$, and $x \underline{*}^{g} x=x \bar{*}^{g} x$ for any $x \in X$ and $g \in G$. By Proposition 4.2 , it is sufficient to prove that $\# \mathrm{Col}_{X}\left(D^{\prime}, \phi^{\prime}\right) \geq \# X$. Here for any $x \in X$ and $g \in G$, we write $x *^{g} x$ for $x \underline{*}^{g} x$ and $x \bar{*}^{g} x$ simply. Then for any $x \in X$, the assignment of elements of $X$ to each semi-arc of $\left(D^{\prime}, \phi^{\prime}\right)$ as shown in Figures 4 and 5 is an $X$-coloring, where each $g_{i}$ represents an element of $G$ in Figure 5. Therefore we have $\# \operatorname{Col}_{X}\left(D^{\prime}, \phi^{\prime}\right) \geq \# X$.

2. It is sufficient to prove that $\# \operatorname{Col}_{X}(O, \psi)=\# X$ when $m=1$. Let $\left(O_{g}, \psi_{g}\right)$ be a $G$ flowed diagram of an $S^{1}$-oriented trivial handlebody-knot of genus $g$. By Theorem 2.1 , we can deform $\left(O_{g}, \psi_{g}\right)$ into the $G$-flowed diagram $\left(O_{g}^{\prime}, \psi_{g}^{\prime}\right)$ depicted in Figure 6 by a finite sequence of Reidemeister moves preserving Y-orientations, where $a_{i} \in G$ for any $i=1, \ldots, g$, and $e$ is the identity of $G$. By Proposition 4.2, it is sufficient 


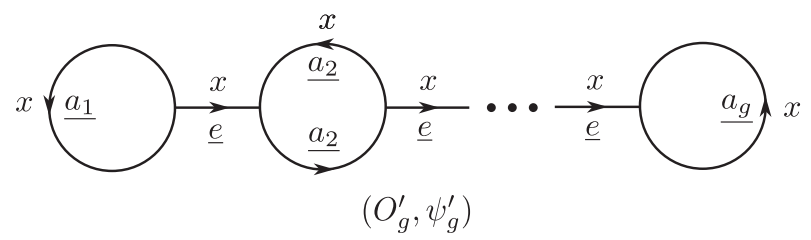

Figure 6. A $G$-flowed diagram $\left(O_{g}^{\prime}, \psi_{g}^{\prime}\right)$ and its $X$-coloring.

to prove that $\# \operatorname{Col}_{X}\left(O_{g}^{\prime}, \psi_{g}^{\prime}\right)=\# X$. For any $x \in X$, the assignment of $x$ to each semi-arc of $\left(O_{g}^{\prime}, \psi_{g}^{\prime}\right)$ as shown in Figure 6 is an $X$-coloring. On the other hand, since any $X$-coloring of $\left(O_{g}^{\prime}, \psi_{g}^{\prime}\right)$ is given by Figure 6 for some $x \in X$, we have $\# \mathrm{Col}_{X}\left(O_{g}^{\prime}, \psi_{g}^{\prime}\right)=\# X$.

\section{Linear relationships for Alexander biquandle colorings.}

For any $\mathbb{Z}_{m}$-flowed diagram $(D, \phi)$ of an $S^{1}$-oriented handlebody-link, we define the Alexander numbering of $(D, \phi)$ by assigning elements of $\mathbb{Z}_{m}$ to each region of $(D, \phi)$ as shown in Figure 7, where the unbounded region is labeled 0. It is an extension of the Alexander numbering of a classical knot diagram [1]. It is easy to see that for any $\mathbb{Z}_{m}$-flowed diagram $(D, \phi)$ of an $S^{1}$-oriented handlebody-link, there uniquely exists the Alexander numbering of $(D, \phi)$. For example, a $\mathbb{Z}_{m}$-flowed diagram of the handlebodyknot $5_{2}[\mathbf{1 0}]$ with the Alexander numbering is depicted in Figure 8. For any semi-arc $\alpha$ of $(D, \phi)$, we denote by $\rho(\alpha)$ the Alexander number of the region which the normal orientation of $\alpha$ points to.

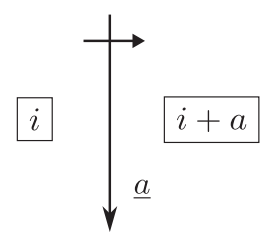

Figure 7. The Alexander numbering of $(D, \phi)$.

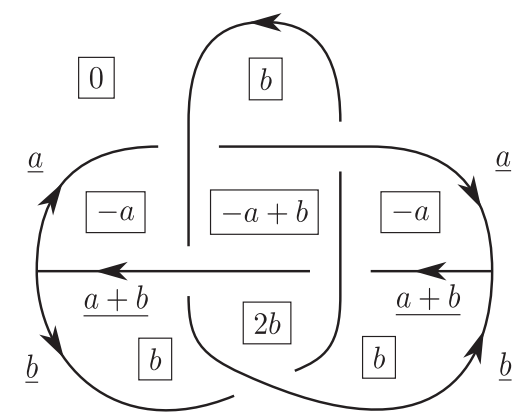

Figure 8. A $\mathbb{Z}_{m}$-flowed diagram of $5_{2}$ with the Alexander numbering. 
In the following, every component of a diagram of any $S^{1}$-oriented handlebody-link may have a crossing at least 1 . Let $(D, \phi)$ be a $\mathbb{Z}_{m}$-flowed diagram of an $S^{1}$-oriented handlebody-link with the Alexander numbering and let $X$ be a $\mathbb{Z}_{m}$-family of Alexander biquandles. We put $C(D, \phi)=\left\{c_{1}, \ldots, c_{n}\right\}$ and $V(D, \phi)=\left\{\tau_{1}, \ldots, \tau_{2 k}\right\}$, where $C(D, \phi)$ and $V(D, \phi)$ are the set of all crossings of $(D, \phi)$ and the one of all vertices of $(D, \phi)$ respectively, where the sign of $\tau_{i}$ is 1 for any $i=1, \ldots, k$ and -1 for any $i=k+1, \ldots, 2 k$. Then we denote by $x_{i}$ each semi-arc of $(D, \phi)$ as shown in Figure 9, which implies $\mathcal{S} \mathcal{A}(D, \phi)=\left\{x_{1}, \ldots, x_{2 n+3 k}\right\}$.
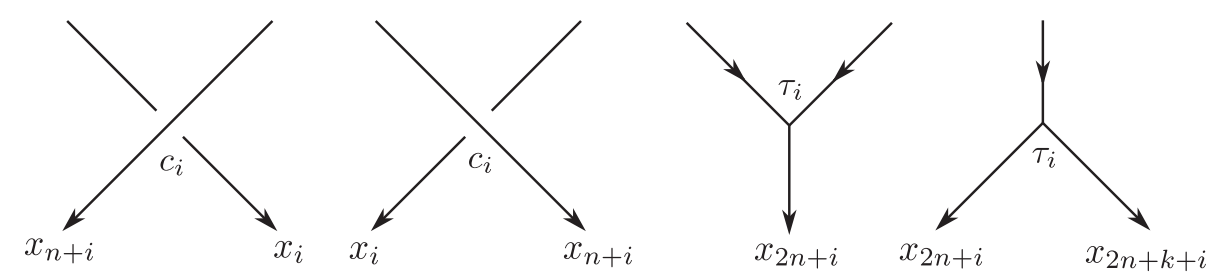

Figure 9. Semi-arcs $x_{i}$ of $(D, \phi)$.

We denote by $u_{i}, v_{i}, v_{i}^{\prime}, w_{i}, \alpha_{i}, \beta_{i}$ and $\gamma_{i}$ the semi-arcs incident to a crossing $c_{i}$ or a vertex $\tau_{i}$ as shown in Figure 10. We put $\phi_{i}:=\phi\left(u_{i}\right)=\phi\left(w_{i}\right), \psi_{i}:=\phi\left(v_{i}\right)=\phi\left(v_{i}^{\prime}\right)$, $\eta_{i}:=\phi\left(\alpha_{i}\right)$ and $\theta_{i}:=\phi\left(\beta_{i}\right)$. We denote by $\epsilon_{c_{i}} \in\{ \pm 1\}$ and $\epsilon_{\tau_{i}} \in\{ \pm 1\}$ the signs of a crossing $c_{i}$ and a vertex $\tau_{i}$ respectively (see Figure 10).
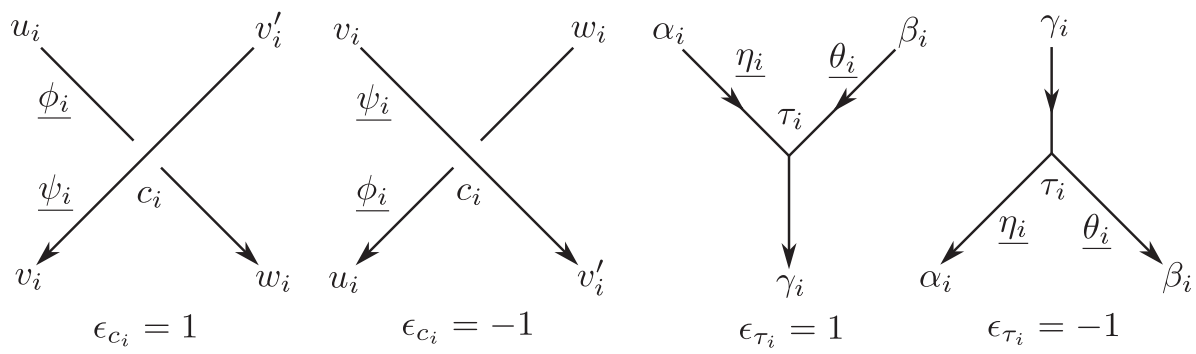

Figure 10. Notations.

For any semi-arcs $y, y^{\prime} \in \mathcal{S} \mathcal{A}(D, \phi)$, we put

$$
\delta\left(y, y^{\prime}\right):= \begin{cases}1 & \left(y=y^{\prime}\right), \\ 0 & \left(y \neq y^{\prime}\right) .\end{cases}
$$

Then we define a matrix $A(D, \phi ; X)=\left(a_{i, j}\right) \in M(2 n+4 k, 2 n+3 k ; X)$ by

$$
a_{i, j}= \begin{cases}\delta\left(u_{i}, x_{j}\right) t^{\psi_{i}}+\delta\left(v_{i}, x_{j}\right)\left(s^{\psi_{i}}-t^{\psi_{i}}\right)-\delta\left(w_{i}, x_{j}\right) & (1 \leq i \leq n), \\ -\delta\left(v_{i-n}, x_{j}\right) s^{\phi_{i-n}}+\delta\left(v_{i-n}^{\prime}, x_{j}\right) & (n+1 \leq i \leq 2 n), \\ \delta\left(\alpha_{i-2 n}, x_{j}\right)-\delta\left(\gamma_{i-2 n}, x_{j}\right) & (2 n+1 \leq i \leq 2 n+2 k), \\ \delta\left(\beta_{i-2 n-2 k}, x_{j}\right)-\delta\left(\gamma_{i-2 n-2 k}, x_{j}\right) s^{\eta_{i-2 n-2 k}} & (2 n+2 k+1 \leq i \leq 2 n+4 k) .\end{cases}
$$


We note that $A(D, \phi ; X)$ is determined up to permuting of rows and columns of the matrix, and it follows that

$$
\operatorname{Col}_{X}(D, \phi)=\left\{\left(\begin{array}{c}
z_{1} \\
z_{2} \\
\vdots \\
z_{2 n+3 k}
\end{array}\right) \in X^{2 n+3 k} \mid A(D, \phi ; X)\left(\begin{array}{c}
z_{1} \\
z_{2} \\
\vdots \\
z_{2 n+3 k}
\end{array}\right)=\mathbf{0}\right\} .
$$

For example, let $(E, \psi)$ be the $\mathbb{Z}_{m}$-flowed diagram of the handlebody-knot depicted in Figure 11. Then we have

$$
A(E, \psi ; X)=\left(\begin{array}{ccccccc}
-1 & 0 & s^{a}-t^{a} & t^{a} & 0 & 0 & 0 \\
0 & -1 & 0 & s^{b}-t^{b} & 0 & t^{b} & 0 \\
0 & 1 & -s^{b} & 0 & 0 & 0 & 0 \\
0 & 0 & 0 & -s^{a} & 0 & 0 & 1 \\
0 & 0 & 1 & 0 & -1 & 0 & 0 \\
0 & 0 & 0 & 0 & -1 & 1 & 0 \\
1 & 0 & 0 & 0 & -s^{a} & 0 & 0 \\
0 & 0 & 0 & 0 & -s^{a} & 0 & 1
\end{array}\right) .
$$

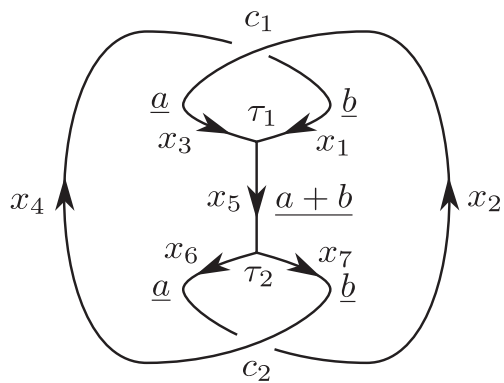

Figure 11. $\quad$ A $\mathbb{Z}_{m}$-flowed diagram $(E, \psi)$.

Then we have the following proposition.

Proposition 5.1. Let $(D, \phi)$ be a $\mathbb{Z}_{m}$-flowed diagram of an $S^{1}$-oriented handlebody-link with the Alexander numbering and let $X$ be a $\mathbb{Z}_{m}$-family of Alexander biquandles. Let $\boldsymbol{a}_{i}$ be the $i$-th row of $A(D, \phi ; X)$. Then it follows that

$$
\begin{aligned}
& \sum_{i=1}^{n} \epsilon_{c_{i}} t^{-\rho\left(w_{i}\right)}\left(s^{\phi_{i}}-t^{\phi_{i}}\right) \boldsymbol{a}_{i}+\sum_{i=1}^{n} \epsilon_{c_{i}} t^{-\rho\left(v_{i}^{\prime}\right)}\left(s^{\psi_{i}}-t^{\psi_{i}}\right) \boldsymbol{a}_{n+i} \\
& +\sum_{i=1}^{2 k} \epsilon_{\tau_{i}} t^{-\rho\left(\alpha_{i}\right)}\left(s^{\eta_{i}}-t^{\eta_{i}}\right) \boldsymbol{a}_{2 n+i}+\sum_{i=1}^{2 k} \epsilon_{\tau_{i}} t^{-\rho\left(\beta_{i}\right)}\left(s^{\theta_{i}}-t^{\theta_{i}}\right) \boldsymbol{a}_{2 n+2 k+i}=\mathbf{0} .
\end{aligned}
$$

Proof. For any semi-arc $y$ incident to a crossing or a vertex $\sigma$, we put 


$$
\epsilon(y ; \sigma):= \begin{cases}1 & \text { if the orientation of } y \text { points to } \sigma \\ -1 & \text { otherwise. }\end{cases}
$$

We set $\left(a_{i, j}\right):=A(D, \phi ; X)$. It is sufficient to prove that for any $j=1,2, \ldots, 2 n+3 k$,

$$
\begin{aligned}
& \sum_{i=1}^{n} \epsilon_{c_{i}} t^{-\rho\left(w_{i}\right)}\left(s^{\phi_{i}}-t^{\phi_{i}}\right) a_{i, j}+\sum_{i=1}^{n} \epsilon_{c_{i}} t^{-\rho\left(v_{i}^{\prime}\right)}\left(s^{\psi_{i}}-t^{\psi_{i}}\right) a_{n+i, j} \\
& +\sum_{i=1}^{2 k} \epsilon_{\tau_{i}} t^{-\rho\left(\alpha_{i}\right)}\left(s^{\eta_{i}}-t^{\eta_{i}}\right) a_{2 n+i, j}+\sum_{i=1}^{2 k} \epsilon_{\tau_{i}} t^{-\rho\left(\beta_{i}\right)}\left(s^{\theta_{i}}-t^{\theta_{i}}\right) a_{2 n+2 k+i, j}=0 .
\end{aligned}
$$

For the first term, we have

$$
\begin{aligned}
& \epsilon_{c_{i}} t^{-\rho\left(w_{i}\right)}\left(s^{\phi_{i}}-t^{\phi_{i}}\right) \delta\left(u_{i}, x_{j}\right) t^{\psi_{i}}=\delta\left(u_{i}, x_{j}\right) \epsilon\left(u_{i} ; c_{i}\right) t^{-\rho\left(u_{i}\right)}\left(s^{\phi\left(u_{i}\right)}-t^{\phi\left(u_{i}\right)}\right), \\
& \epsilon_{c_{i}} t^{-\rho\left(w_{i}\right)}\left(s^{\phi_{i}}-t^{\phi_{i}}\right) \delta\left(v_{i}, x_{j}\right)\left(s^{\psi_{i}}-t^{\psi_{i}}\right) \\
& =\epsilon_{c_{i}} t^{-\rho\left(w_{i}\right)} s^{\phi_{i}} \delta\left(v_{i}, x_{j}\right)\left(s^{\psi_{i}}-t^{\psi_{i}}\right)-\epsilon_{c_{i}} t^{-\rho\left(w_{i}\right)} t^{\phi_{i}} \delta\left(v_{i}, x_{j}\right)\left(s^{\psi_{i}}-t^{\psi_{i}}\right) \\
& =\epsilon_{c_{i}} t^{-\rho\left(w_{i}\right)} \delta\left(v_{i}, x_{j}\right)\left(s^{\psi_{i}}-t^{\psi_{i}}\right) s^{\phi_{i}}+\delta\left(v_{i}, x_{j}\right) \epsilon\left(v_{i} ; c_{i}\right) t^{-\rho\left(v_{i}\right)}\left(s^{\phi\left(v_{i}\right)}-t^{\phi\left(v_{i}\right)}\right), \\
& \epsilon_{c_{i}} t^{-\rho\left(w_{i}\right)}\left(s^{\phi_{i}}-t^{\phi_{i}}\right)\left(-\delta\left(w_{i}, x_{j}\right)\right)=\delta\left(w_{i}, x_{j}\right) \epsilon\left(w_{i} ; c_{i}\right) t^{-\rho\left(w_{i}\right)}\left(s^{\phi\left(w_{i}\right)}-t^{\phi\left(w_{i}\right)}\right) .
\end{aligned}
$$

For the second term, we have

$$
\begin{aligned}
& \epsilon_{c_{i}} t^{-\rho\left(v_{i}^{\prime}\right)}\left(s^{\psi_{i}}-t^{\psi_{i}}\right)\left(-\delta\left(v_{i}, x_{j}\right) s^{\phi_{i}}\right)=-\epsilon_{c_{i}} t^{-\rho\left(v_{i}^{\prime}\right)} \delta\left(v_{i}, x_{j}\right)\left(s^{\psi_{i}}-t^{\psi_{i}}\right) s^{\phi_{i}}, \\
& \epsilon_{c_{i}} t^{-\rho\left(v_{i}^{\prime}\right)}\left(s^{\psi_{i}}-t^{\psi_{i}}\right) \delta\left(v_{i}^{\prime}, x_{j}\right)=\delta\left(v_{i}^{\prime}, x_{j}\right) \epsilon\left(v_{i}^{\prime} ; c_{i}\right) t^{-\rho\left(v_{i}^{\prime}\right)}\left(s^{\phi\left(v_{i}^{\prime}\right)}-t^{\phi\left(v_{i}^{\prime}\right)}\right) .
\end{aligned}
$$

For the third term, we have

$$
\begin{aligned}
& \epsilon_{\tau_{i}} t^{-\rho\left(\alpha_{i}\right)}\left(s^{\eta_{i}}-t^{\eta_{i}}\right) \delta\left(\alpha_{i}, x_{j}\right)=\delta\left(\alpha_{i}, x_{j}\right) \epsilon\left(\alpha_{i} ; \tau_{i}\right) t^{-\rho\left(\alpha_{i}\right)}\left(s^{\phi\left(\alpha_{i}\right)}-t^{\phi\left(\alpha_{i}\right)}\right), \\
& \epsilon_{\tau_{i}} t^{-\rho\left(\alpha_{i}\right)}\left(s^{\eta_{i}}-t^{\eta_{i}}\right)\left(-\delta\left(\gamma_{i}, x_{j}\right)\right)=\delta\left(\gamma_{i}, x_{j}\right) \epsilon\left(\gamma_{i} ; \tau_{i}\right) t^{-\rho\left(\gamma_{i}\right)} t^{\theta_{i}}\left(s^{\eta_{i}}-t^{\eta_{i}}\right) .
\end{aligned}
$$

For the last term, we have

$$
\begin{aligned}
& \epsilon_{\tau_{i}} t^{-\rho\left(\beta_{i}\right)}\left(s^{\theta_{i}}-t^{\theta_{i}}\right) \delta\left(\beta_{i}, x_{j}\right)=\delta\left(\beta_{i}, x_{j}\right) \epsilon\left(\beta_{i} ; \tau_{i}\right) t^{-\rho\left(\beta_{i}\right)}\left(s^{\phi\left(\beta_{i}\right)}-t^{\phi\left(\beta_{i}\right)}\right), \\
& \epsilon_{\tau_{i}} t^{-\rho\left(\beta_{i}\right)}\left(s^{\theta_{i}}-t^{\theta_{i}}\right)\left(-\delta\left(\gamma_{i}, x_{j}\right) s^{\eta_{i}}\right)=\delta\left(\gamma_{i}, x_{j}\right) \epsilon\left(\gamma_{i} ; \tau_{i}\right) t^{-\rho\left(\gamma_{i}\right)}\left(s^{\theta_{i}}-t^{\theta_{i}}\right) s^{\eta_{i}} .
\end{aligned}
$$

We note that

$$
\begin{aligned}
& (1)+(2)=\delta\left(v_{i}, x_{j}\right) \epsilon\left(v_{i} ; c_{i}\right) t^{-\rho\left(v_{i}\right)}\left(s^{\phi\left(v_{i}\right)}-t^{\phi\left(v_{i}\right)}\right), \\
& (3)+(4)=\delta\left(\gamma_{i}, x_{j}\right) \epsilon\left(\gamma_{i} ; \tau_{i}\right) t^{-\rho\left(\gamma_{i}\right)}\left(s^{\phi\left(\gamma_{i}\right)}-t^{\phi\left(\gamma_{i}\right)}\right) .
\end{aligned}
$$

Therefore for any $j=1,2, \ldots, 2 n+3 k$, it follows that

$$
\sum_{i=1}^{n} \epsilon_{c_{i}} t^{-\rho\left(w_{i}\right)}\left(s^{\phi_{i}}-t^{\phi_{i}}\right) a_{i, j}+\sum_{i=1}^{n} \epsilon_{c_{i}} t^{-\rho\left(v_{i}^{\prime}\right)}\left(s^{\psi_{i}}-t^{\psi_{i}}\right) a_{n+i, j}
$$




$$
\begin{aligned}
& +\sum_{i=1}^{2 k} \epsilon_{\tau_{i}} t^{-\rho\left(\alpha_{i}\right)}\left(s^{\eta_{i}}-t^{\eta_{i}}\right) a_{2 n+i, j}+\sum_{i=1}^{2 k} \epsilon_{\tau_{i}} t^{-\rho\left(\beta_{i}\right)}\left(s^{\theta_{i}}-t^{\theta_{i}}\right) a_{2 n+2 k+i, j} \\
& =\sum_{i=1}^{n}\left(\delta\left(u_{i}, x_{j}\right) \epsilon\left(u_{i} ; c_{i}\right) t^{-\rho\left(u_{i}\right)}\left(s^{\phi\left(u_{i}\right)}-t^{\phi\left(u_{i}\right)}\right)\right. \\
& \quad+\delta\left(v_{i}, x_{j}\right) \epsilon\left(v_{i} ; c_{i}\right) t^{-\rho\left(v_{i}\right)}\left(s^{\phi\left(v_{i}\right)}-t^{\phi\left(v_{i}\right)}\right) \\
& \quad+\delta\left(v_{i}^{\prime}, x_{j}\right) \epsilon\left(v_{i}^{\prime} ; c_{i}\right) t^{-\rho\left(v_{i}^{\prime}\right)}\left(s^{\phi\left(v_{i}^{\prime}\right)}-t^{\phi\left(v_{i}^{\prime}\right)}\right) \\
& \left.\quad+\delta\left(w_{i}, x_{j}\right) \epsilon\left(w_{i} ; c_{i}\right) t^{-\rho\left(w_{i}\right)}\left(s^{\phi\left(w_{i}\right)}-t^{\phi\left(w_{i}\right)}\right)\right) \\
& \quad+\sum_{i=1}^{2 k}\left(\delta\left(\alpha_{i}, x_{j}\right) \epsilon\left(\alpha_{i} ; \tau_{i}\right) t^{-\rho\left(\alpha_{i}\right)}\left(s^{\phi\left(\alpha_{i}\right)}-t^{\phi\left(\alpha_{i}\right)}\right)\right. \\
& \quad+\delta\left(\beta_{i}, x_{j}\right) \epsilon\left(\beta_{i} ; \tau_{i}\right) t^{-\rho\left(\beta_{i}\right)}\left(s^{\phi\left(\beta_{i}\right)}-t^{\phi\left(\beta_{i}\right)}\right) \\
& \left.\quad+\delta\left(\gamma_{i}, x_{j}\right) \epsilon\left(\gamma_{i} ; \tau_{i}\right) t^{-\rho\left(\gamma_{i}\right)}\left(s^{\phi\left(\gamma_{i}\right)}-t^{\phi\left(\gamma_{i}\right)}\right)\right) \\
& =t^{-\rho\left(x_{j}\right)}\left(s^{\phi\left(x_{j}\right)}-t^{\phi\left(x_{j}\right)}\right)-t^{-\rho\left(x_{j}\right)}\left(s^{\phi\left(x_{j}\right)}-t^{\phi\left(x_{j}\right)}\right) \\
& =0 .
\end{aligned}
$$

Let $X$ be an Alexander biquandle and let $m=$ type $X$. Then $X$ is also a $\mathbb{Z}_{m}$-family of Alexander biquandles. Let $D$ be an oriented classical link diagram. We can regard $D$ as a $\mathbb{Z}_{m}$-flowed diagram $\left(D, \phi_{(1)}\right)$ of an $S^{1}$-oriented handlebody-link whose components are of genus 1 , where $\phi_{(1)}$ is the constant map to 1 . Hence we can regard an $X$-coloring of $D$ as an $X$-coloring of $\left(D, \phi_{(1)}\right)$. We define a matrix $A(D ; X) \in M(2 n, 2 n ; X)$ by $A(D ; X)=A\left(D, \phi_{(1)} ; X\right)$, where $n$ is the number of crossings of $D$. Then the set of all $X$-colorings of $D$, denoted by $\operatorname{Col}_{X}(D)$, is given by

$$
\operatorname{Col}_{X}(D)=\left\{\left(\begin{array}{c}
z_{1} \\
z_{2} \\
\vdots \\
z_{2 n}
\end{array}\right) \in X^{2 n} \mid A(D ; X)\left(\begin{array}{c}
z_{1} \\
z_{2} \\
\vdots \\
z_{2 n}
\end{array}\right)=\mathbf{0}\right\} .
$$

Therefore we obtain the following corollary.

Corollary 5.2. Let $D$ be a diagram of an oriented classical link with the Alexander numbering and let $X$ be an Alexander biquandle. Let $\boldsymbol{a}_{i}$ be the $i$-th row of $A(D ; X)$. Then it follows that

$$
\sum_{i=1}^{n} \epsilon_{c_{i}} t^{-\rho\left(w_{i}\right)}(s-t) \boldsymbol{a}_{i}+\sum_{i=1}^{n} \epsilon_{c_{i}} t^{-\rho\left(v_{i}^{\prime}\right)}(s-t) \boldsymbol{a}_{n+i}=\mathbf{0} .
$$

\section{Main theorem.}

In this section, we give lower bounds for the Gordian distance and the unknotting number of $S^{1}$-oriented handlebody-knots.

TheOREm 6.1. Let $H_{i}$ be an $S^{1}$-oriented handlebody-knot of genus $g$ and let $D_{i}$ be 
a diagram of $H_{i}(i=1,2)$. Let $X=\mathbb{Z}_{p}\left[t^{ \pm 1}\right] /(f(t))$ which is a $\mathbb{Z}_{m}$-family of Alexander biquandles, where $p$ is a prime number, $s \in \mathbb{Z}_{p}\left[t^{ \pm 1}\right]$ and $f(t) \in \mathbb{Z}_{p}\left[t^{ \pm 1}\right]$ is an irreducible polynomial. Then it follows that

$$
\max _{\phi_{1} \in \operatorname{Flow}\left(D_{1} ; \mathbb{Z}_{m}\right)} \min _{\substack{\phi_{2} \in \operatorname{Flow}\left(D_{2} ; \mathbb{Z}_{m}\right) \\ \operatorname{gcd} \phi_{1}=\operatorname{gcd} \phi_{2}}}\left|\operatorname{dim} \operatorname{Col}_{X}\left(D_{1}, \phi_{1}\right)-\operatorname{dim} \operatorname{Col}_{X}\left(D_{2}, \phi_{2}\right)\right| \leq d\left(H_{1}, H_{2}\right) .
$$

Proof. Let $(D, \phi)$ be a $\mathbb{Z}_{m}$-flowed diagram of an $S^{1}$-oriented handlebody-knot and let $C(D, \phi)=\left\{c_{1}, \ldots, c_{n}\right\}$ and $V(D, \phi)=\left\{\tau_{1}, \ldots, \tau_{2 k}\right\}$. Let $(\bar{D}, \bar{\phi})$ be the $\mathbb{Z}_{m}$-flowed diagram of an $S^{1}$-oriented handlebody-knot which is obtained from $(D, \phi)$ by the crossing change at $c_{1}$ and let $C(\bar{D}, \bar{\phi})=\left\{\bar{c}_{1}, \ldots, \bar{c}_{n}\right\}$ and $V(\bar{D}, \bar{\phi})=\left\{\bar{\tau}_{1}, \ldots, \bar{\tau}_{2 k}\right\}$, where $\bar{\phi}, \bar{c}_{i}$ and $\bar{\tau}_{i}$ originate from $\phi, c_{i}$ and $\tau_{i}$ naturally and respectively (see Figure 12). In the following, we show that

$$
\left|\operatorname{dim} \operatorname{Col}_{X}(D, \phi)-\operatorname{dim} \operatorname{Col}_{X}(\bar{D}, \bar{\phi})\right| \leq 1
$$

that is,

$$
|\operatorname{rank} A(D, \phi ; X)-\operatorname{rank} A(\bar{D}, \bar{\phi} ; X)| \leq 1
$$
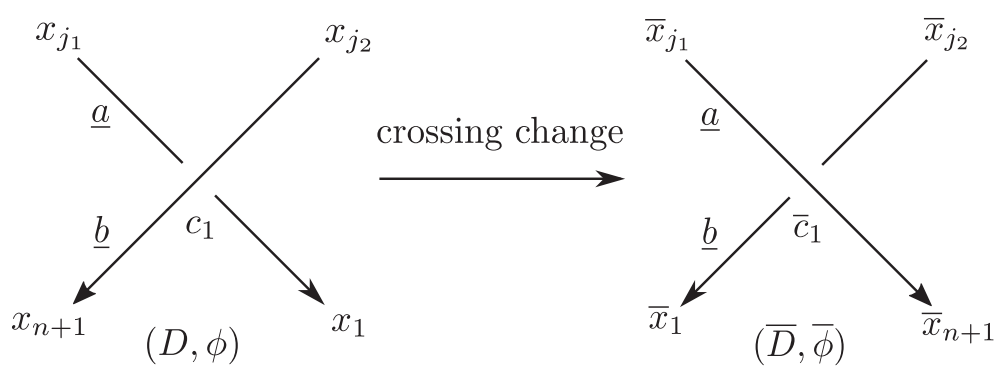

Figure 12. The crossing change at $c_{1}$.

We may assume that $c_{1}$ is a positive crossing and $\bar{c}_{1}$ is a negative crossing. We denote by $\bar{x}_{i}$ each semi-arc of $(\bar{D}, \bar{\phi})$ in the same way as in Figure 9 with respect to $\bar{c}_{i}$ or $\bar{\tau}_{i}$, and so are $\bar{v}_{i}^{\prime}, \bar{w}_{i}, \bar{\alpha}_{i}, \bar{\beta}_{i}, \bar{\phi}_{i}, \bar{\psi}_{i}, \bar{\eta}_{i}, \bar{\theta}_{i}, \bar{\epsilon}_{c_{i}}$ and $\bar{\epsilon}_{\tau_{i}}$ (see Figure 10). We denote by $x_{j_{1}}$ and $x_{j_{2}}$ the semi-arcs which point to the crossing $c_{1}$ of $(D, \phi)$ as shown in Figure 12, and we put $a:=\phi_{1}=\bar{\psi}_{1}$ and $b:=\psi_{1}=\bar{\phi}_{1}$. We note that $\operatorname{Col}_{X}(D, \phi)$ and $\operatorname{Col}_{X}(\bar{D}, \bar{\phi})$ are vector spaces over $X$ since $X$ is a $\mathbb{Z}_{m}$-family of Alexander biquandles and a field.

Let $\boldsymbol{a}_{i}, \overline{\boldsymbol{a}}_{i}$ and $\hat{\boldsymbol{a}}_{i}$ be the $i$-th rows of $A(D, \phi ; X), A(\bar{D}, \bar{\phi} ; X)$ and $\hat{A}(\bar{D}, \bar{\phi} ; X)$ respectively, where $\hat{A}(\bar{D}, \bar{\phi} ; X)$ is the matrix obtained by permuting the first column and the $(n+1)$-th column of $A(\bar{D}, \bar{\phi} ; X)$. We put $\left(a_{i, j}\right):=A(D, \phi ; X),\left(\bar{a}_{i, j}\right):=A(\bar{D}, \bar{\phi} ; X)$ and $\left(\hat{a}_{i, j}\right):=\hat{A}(\bar{D}, \bar{\phi} ; X)$. Then we have $\boldsymbol{a}_{i}=\hat{\boldsymbol{a}}_{i}$ when $i \neq 1, n+1$. We note that $\operatorname{rank} A(\bar{D}, \bar{\phi} ; X)=\operatorname{rank} \hat{A}(\bar{D}, \bar{\phi} ; X)$ and

$$
\boldsymbol{a}_{1}=\left(-1,0, \ldots, 0, t^{b}, 0, \ldots, 0, s^{b}-t^{b}, 0, \ldots, 0\right),
$$




$$
\begin{aligned}
& \boldsymbol{a}_{n+1}=\left(0, \ldots, 0, \stackrel{j_{2}}{\vee}, 0, \ldots, 0,-s^{a}, 0, \ldots, 0\right), \\
& \overline{\boldsymbol{a}}_{1}=\left(t^{a}, 0, \ldots, 0, s^{a} \stackrel{j_{1}}{\vee} t^{a}, 0, \ldots, 0, \stackrel{j_{2}}{\vee}-1,0, \ldots, 0\right), \\
& \overline{\boldsymbol{a}}_{n+1}=\left(0, \ldots, 0,-s^{b}, 0, \ldots, 0, \stackrel{j_{1}}{\vee} \mathbb{v}^{\vee}, 0, \ldots, 0\right), \\
& \hat{\boldsymbol{a}}_{1}=\left(0, \ldots, 0, s^{a} \stackrel{\stackrel{j_{1}}{\vee}}{-} t^{a}, 0, \ldots, \stackrel{\stackrel{j_{2}}{\vee}}{\stackrel{\vee}{1}}, \stackrel{\stackrel{n+1}{\vee}}{ }, 0, \ldots, 0, t^{a}, 0, \ldots, 0\right), \\
& j_{1} \\
& \hat{\boldsymbol{a}}_{n+1}=\left(1,0, \ldots, 0,-s^{b}, 0, \ldots, 0\right) .
\end{aligned}
$$

By Proposition 5.1, we obtain

$$
\begin{aligned}
& \sum_{i=1}^{n} \epsilon_{c_{i}} t^{-\rho\left(w_{i}\right)}\left(s^{\phi_{i}}-t^{\phi_{i}}\right) \boldsymbol{a}_{i}+\sum_{i=1}^{n} \epsilon_{c_{i}} t^{-\rho\left(v_{i}^{\prime}\right)}\left(s^{\psi_{i}}-t^{\psi_{i}}\right) \boldsymbol{a}_{n+i} \\
& +\sum_{i=1}^{2 k} \epsilon_{\tau_{i}} t^{-\rho\left(\alpha_{i}\right)}\left(s^{\eta_{i}}-t^{\eta_{i}}\right) \boldsymbol{a}_{2 n+i}+\sum_{i=1}^{2 k} \epsilon_{\tau_{i}} t^{-\rho\left(\beta_{i}\right)}\left(s^{\theta_{i}}-t^{\theta_{i}}\right) \boldsymbol{a}_{2 n+2 k+i}=\mathbf{0}
\end{aligned}
$$

and

$$
\begin{aligned}
& \sum_{i=1}^{n} \bar{\epsilon}_{c_{i}} t^{-\rho\left(\bar{w}_{i}\right)}\left(s^{\bar{\phi}_{i}}-t^{\bar{\phi}_{i}}\right) \overline{\boldsymbol{a}}_{i}+\sum_{i=1}^{n} \bar{\epsilon}_{c_{i}} t^{-\rho\left(\bar{v}_{i}^{\prime}\right)}\left(s^{\bar{\psi}_{i}}-t^{\bar{\psi}_{i}}\right) \overline{\boldsymbol{a}}_{n+i} \\
& \quad+\sum_{i=1}^{2 k} \bar{\epsilon}_{\tau_{i}} t^{-\rho\left(\bar{\alpha}_{i}\right)}\left(s^{\bar{\eta}_{i}}-t^{\bar{\eta}_{i}}\right) \overline{\boldsymbol{a}}_{2 n+i}+\sum_{i=1}^{2 k} \bar{\epsilon}_{\tau_{i}} t^{-\rho\left(\bar{\beta}_{i}\right)}\left(s^{\bar{\theta}_{i}}-t^{\bar{\theta}_{i}}\right) \overline{\boldsymbol{a}}_{2 n+2 k+i} \\
& =\sum_{i=1}^{n} \bar{\epsilon}_{c_{i}} t^{-\rho\left(\bar{w}_{i}\right)}\left(s^{\bar{\phi}_{i}}-t^{\bar{\phi}_{i}}\right) \hat{\boldsymbol{a}}_{i}+\sum_{i=1}^{n} \bar{\epsilon}_{c_{i}} t^{-\rho\left(\bar{v}_{i}^{\prime}\right)}\left(s^{\bar{\psi}_{i}}-t^{\bar{\psi}_{i}}\right) \hat{\boldsymbol{a}}_{n+i} \\
& \quad+\sum_{i=1}^{2 k} \bar{\epsilon}_{\tau_{i}} t^{-\rho\left(\bar{\alpha}_{i}\right)}\left(s^{\bar{\eta}_{i}}-t^{\bar{\eta}_{i}}\right) \hat{\boldsymbol{a}}_{2 n+i}+\sum_{i=1}^{2 k} \bar{\epsilon}_{\tau_{i}} t^{-\rho\left(\bar{\beta}_{i}\right)}\left(s^{\bar{\theta}_{i}}-t^{\bar{\theta}_{i}}\right) \hat{\boldsymbol{a}}_{2 n+2 k+i}=\mathbf{0 .} .
\end{aligned}
$$

If $\epsilon_{c_{1}} t^{-\rho\left(w_{1}\right)}\left(s^{\phi_{1}}-t^{\phi_{1}}\right)=0$, we have $s^{\phi_{1}}-t^{\phi_{1}}=s^{a}-t^{a}=0$, which implies that $\boldsymbol{a}_{n+1}=-\hat{\boldsymbol{a}}_{1}$. Hence it follows that

$|\operatorname{rank} A(D, \phi ; X)-\operatorname{rank} A(\bar{D}, \bar{\phi} ; X)|=|\operatorname{rank} A(D, \phi ; X)-\operatorname{rank} \hat{A}(\bar{D}, \bar{\phi} ; X)| \leq 1$.

If $\bar{\epsilon}_{c_{1}} t^{-\rho\left(\bar{w}_{1}\right)}\left(s^{\bar{\phi}_{1}}-t^{\bar{\phi}_{1}}\right)=0$, we have $s^{\bar{\phi}_{1}}-t^{\bar{\phi}_{1}}=s^{b}-t^{b}=0$, which implies that $\boldsymbol{a}_{1}=$ $-\hat{\boldsymbol{a}}_{n+1}$. Hence it follows that

$|\operatorname{rank} A(D, \phi ; X)-\operatorname{rank} A(\bar{D}, \bar{\phi} ; X)|=|\operatorname{rank} A(D, \phi ; X)-\operatorname{rank} \hat{A}(\bar{D}, \bar{\phi} ; X)| \leq 1$.

If $\epsilon_{c_{1}} t^{-\rho\left(w_{1}\right)}\left(s^{\phi_{1}}-t^{\phi_{1}}\right) \neq 0$ and $\bar{\epsilon}_{c_{1}} t^{-\rho\left(\bar{w}_{1}\right)}\left(s^{\bar{\phi}_{1}}-t^{\bar{\phi}_{1}}\right) \neq 0$, we can represent $\boldsymbol{a}_{1}$ and $\overline{\boldsymbol{a}}_{1}$ as linear combinations of $\boldsymbol{a}_{2}, \ldots, \boldsymbol{a}_{2 n+4 k}$ and $\overline{\boldsymbol{a}}_{2}, \ldots, \overline{\boldsymbol{a}}_{2 n+4 k}$ respectively. Hence it 
follows that

$$
\operatorname{rank} A(D, \phi ; X)=\operatorname{rank}\left(\begin{array}{c}
\boldsymbol{a}_{2} \\
\vdots \\
\boldsymbol{a}_{2 n+4 k}
\end{array}\right), \operatorname{rank} A(\bar{D}, \bar{\phi} ; X)=\operatorname{rank}\left(\begin{array}{c}
\overline{\boldsymbol{a}}_{2} \\
\vdots \\
\overline{\boldsymbol{a}}_{2 n+4 k}
\end{array}\right),
$$

which implies that

$$
\begin{aligned}
|\operatorname{rank} A(D, \phi ; X)-\operatorname{rank} A(\bar{D}, \bar{\phi} ; X)| & =\left|\operatorname{rank}\left(\begin{array}{c}
\boldsymbol{a}_{2} \\
\vdots \\
\boldsymbol{a}_{2 n+4 k}
\end{array}\right)-\operatorname{rank}\left(\begin{array}{c}
\overline{\boldsymbol{a}}_{2} \\
\vdots \\
\overline{\boldsymbol{a}}_{2 n+4 k}
\end{array}\right)\right| \\
& =\left|\operatorname{rank}\left(\begin{array}{c}
\boldsymbol{a}_{2} \\
\vdots \\
\boldsymbol{a}_{2 n+4 k}
\end{array}\right)-\operatorname{rank}\left(\begin{array}{c}
\hat{\boldsymbol{a}}_{2} \\
\vdots \\
\hat{\boldsymbol{a}}_{2 n+4 k}
\end{array}\right)\right| \\
& \leq 1 .
\end{aligned}
$$

Consequently, if we can deform $H_{1}$ into $H_{2}$ by crossing changes at $l$ crossings, then for any $\mathbb{Z}_{m}$-flowed diagram $\left(D_{1}, \phi_{1}\right)$ of $H_{1}$, there exists a $\mathbb{Z}_{m}$-flowed diagram $\left(D_{2}, \phi_{2}\right)$ of $H_{2}$ satisfying $\operatorname{gcd} \phi_{1}=\operatorname{gcd} \phi_{2}$ and

$$
\left|\operatorname{dim} \operatorname{Col}_{X}\left(D_{1}, \phi_{1}\right)-\operatorname{dim} \operatorname{Col}_{X}\left(D_{2}, \phi_{2}\right)\right| \leq l
$$

by Lemma 4.1. Therefore it follows that

$$
\max _{\phi_{1} \in \operatorname{Flow}\left(D_{1} ; \mathbb{Z}_{m}\right)} \min _{\substack{\phi_{2} \in \operatorname{Flow}\left(D_{2} ; \mathbb{Z}_{m}\right) \\ \operatorname{gcd} \phi_{1}=\operatorname{gcd} \phi_{2}}}\left|\operatorname{dim} \operatorname{Col}_{X}\left(D_{1}, \phi_{1}\right)-\operatorname{dim} \operatorname{Col}_{X}\left(D_{2}, \phi_{2}\right)\right| \leq d\left(H_{1}, H_{2}\right) .
$$

By Proposition 4.3 and Theorem 6.1, the following corollary holds immediately.

Corollary 6.2. Let $H$ be an $S^{1}$-oriented handlebody-knot and let $D$ be a diagram of $H$. Let $X=\mathbb{Z}_{p}\left[t^{ \pm 1}\right] /(f(t))$ which is a $\mathbb{Z}_{m}$-family of Alexander biquandles, where $p$ is a prime number, $s \in \mathbb{Z}_{p}\left[t^{ \pm 1}\right]$ and $f(t) \in \mathbb{Z}_{p}\left[t^{ \pm 1}\right]$ is an irreducible polynomial. Then it follows that

$$
\max _{\phi \in \operatorname{Flow}\left(D ; \mathbb{Z}_{m}\right)} \operatorname{dim} \operatorname{Col}_{X}(D, \phi)-1 \leq u(H)
$$

\section{Examples.}

In this section, we give some examples. In Example 7.1, we give a handlebody-knot with unknotting number 2 , and in Remark 7.2 , we note that it can not be obtained by using Alexander quandle colorings with $\mathbb{Z}_{2}, \mathbb{Z}_{3}$-flows introduced in [12]. In Example 7.3, we give three handlebody-knots with unknotting number $n$ for any $n \in \mathbb{Z}_{>0}$. In Example 7.4 , we give two handlebody-knots with their Gordian distance $n$ for any $n \in \mathbb{Z}_{>0}$.

EXAMPLE 7.1. Let $H$ be the handlebody-knot represented by the $\mathbb{Z}_{10}$-flowed diagram $(D, \phi)$ depicted in Figure 13. Then we show that $u(H)=2$. 


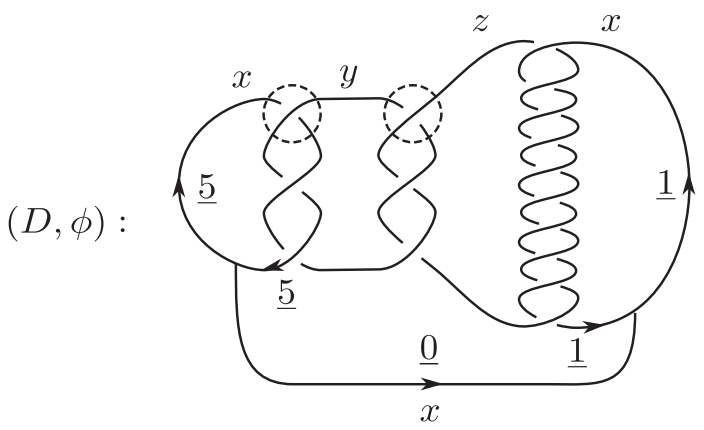

Figure 13. A $\mathbb{Z}_{10}$-flowed diagram $(D, \phi)$ of $H$.

Let $s=1 \in \mathbb{Z}_{3}\left[t^{ \pm 1}\right]$ and let $f(t)=t^{4}+2 t^{3}+t^{2}+2 t+1 \in \mathbb{Z}_{3}\left[t^{ \pm 1}\right]$, which is an irreducible polynomial. Then $X:=\mathbb{Z}_{3}\left[t^{ \pm 1}\right] /(f(t))$ is a $\mathbb{Z}_{10}$-family of Alexander biquandles. Then for any $x, y, z \in X$, the assignment of them to each semi-arc of $(D, \phi)$ as shown in Figure 13 is an $X$-coloring of $(D, \phi)$, which implies $\operatorname{dim} \operatorname{Col}_{X}(D, \phi) \geq 3$. By Corollary 6.2 , we obtain $2 \leq u(H)$. On the other hand, we can deform $H$ into a trivial handlebody-knot by the crossing changes at two crossings surrounded by dotted circles depicted in Figure 13. Therefore it follows that $u(H)=2$.

REMARK 7.2. We show that the result in Example 7.1 can not be obtained by using Alexander quandle colorings with $\mathbb{Z}_{2}, \mathbb{Z}_{3}$-flows introduced in [12].

Let $H$ be the handlebody-knot represented by the $\mathbb{Z}_{m}$-flowed diagram $(D, \phi(a, b))$ depicted in Figure 14 for any $m=2,3$ and $a, b \in \mathbb{Z}_{m}$. Let $p$ be a prime number, $s=1 \in$ $\mathbb{Z}_{p}\left[t^{ \pm 1}\right], f(t)$ be an irreducible polynomial in $\mathbb{Z}_{p}\left[t^{ \pm 1}\right]$ and let $X=\mathbb{Z}_{p}\left[t^{ \pm 1}\right] /(f(t))$ which is a $\mathbb{Z}_{m}$-family of Alexander (bi)quandles. We note that $\operatorname{Col}_{X}(D, \phi(a, b))$ is generated by $x, y, z \in X$ as shown in Figure 14 for any $m=2,3$ and $a, b \in \mathbb{Z}_{m}$. If $(a, b)=(1,0), x, y$ and $z$ need to satisfy the following relations:

$$
\begin{aligned}
& \left(t^{2}-t+1\right) x-\left(t^{2}-t+1\right) y=0, \\
& -t\left(t^{2}-t+1\right) x+t^{-1}(t+1)(t-1)\left(t^{2}-t+1\right) y+t^{-1}\left(t^{2}-t+1\right) z=0, \\
& -t^{-1}(t-1)\left(t^{2}-t+1\right) x+t^{-2}\left(t^{2}-t-1\right)\left(t^{2}-t+1\right) y+t^{-2}\left(t^{2}-t+1\right) z=0, \\
& \left(\left(t^{3}+t^{2}-1\right)\left(t^{2}-t+1\right)-t\right) x-\left(\left(t^{3}+t^{2}-1\right)\left(t^{2}-t+1\right)-t\right) z=0,
\end{aligned}
$$

that is,

$$
M\left(\begin{array}{l}
x \\
y \\
z
\end{array}\right)=\left(\begin{array}{l}
0 \\
0 \\
0 \\
0
\end{array}\right)
$$

where

$$
M=\left(\begin{array}{ccc}
t^{2}-t+1 & -\left(t^{2}-t+1\right) & 0 \\
-t\left(t^{2}-t+1\right) & t^{-1}(t+1)(t-1)\left(t^{2}-t+1\right) & t^{-1}\left(t^{2}-t+1\right) \\
-t^{-1}(t-1)\left(t^{2}-t+1\right) & t^{-2}\left(t^{2}-t-1\right)\left(t^{2}-t+1\right) & t^{-2}\left(t^{2}-t+1\right) \\
\left(t^{3}+t^{2}-1\right)\left(t^{2}-t+1\right)-t & 0 & -\left(t^{3}+t^{2}-1\right)\left(t^{2}-t+1\right)+t
\end{array}\right) .
$$




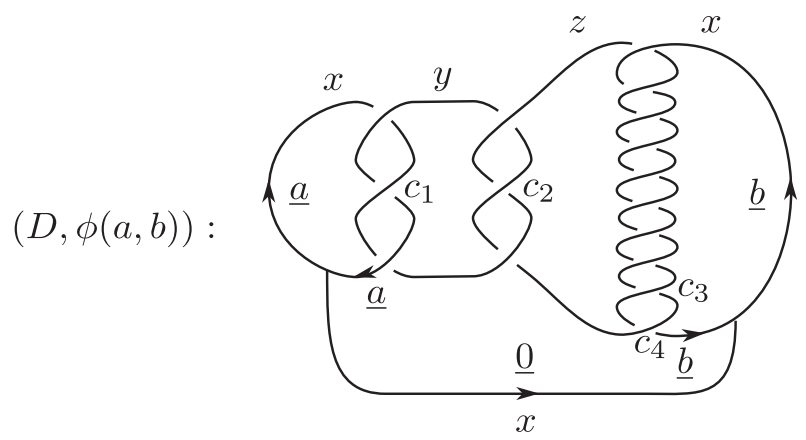

Figure 14. A $\mathbb{Z}_{m}$-flowed diagram $(D, \phi(a, b))$ of $H$.

These relations are obtained from crossings $c_{1}, c_{2}, c_{3}$ and $c_{4}$ as shown in Figure 14. When $t^{2}-t+1 \neq 0$ in $X$, it is clearly that rank $M \geq 1$. When $t^{2}-t+1=0$ in $X$, we have

$$
M=\left(\begin{array}{ccc}
0 & 0 & 0 \\
0 & 0 & 0 \\
0 & 0 & 0 \\
-t & 0 & t
\end{array}\right),
$$

which implies that $\operatorname{rank} M=1$. Hence we have $\operatorname{dim} \operatorname{Col}_{X}(D, \phi(1,0))=3-\operatorname{rank} M \leq 2$. Therefore we can not obtain $2 \leq u(H)$.

We can prove the remaining cases in the same way.

ExAmple 7.3. Let $A_{n}, B_{n}$ and $C_{n}$ be the handlebody-knots represented by the $\mathbb{Z}_{8}$-flowed diagram $\left(D_{A_{n}}, \phi_{A_{n}}\right)$, the $\mathbb{Z}_{24}$-flowed diagram $\left(D_{B_{n}}, \phi_{B_{n}}\right)$ and the $\mathbb{Z}_{8}$-flowed diagram $\left(D_{C_{n}}, \phi_{C_{n}}\right)$ depicted in Figures 15,16 and 17 respectively for any $n \in \mathbb{Z}_{>0}$. Then we show that $u\left(A_{n}\right)=u\left(B_{n}\right)=u\left(C_{n}\right)=n$.

1. Let $s=t+1 \in \mathbb{Z}_{3}\left[t^{ \pm 1}\right]$ and let $f(t)=t^{2}+t+2 \in \mathbb{Z}_{3}\left[t^{ \pm 1}\right]$, which is an irreducible polynomial. Then $X:=\mathbb{Z}_{3}\left[t^{ \pm 1}\right] /(f(t))$ is a $\mathbb{Z}_{8}$-family of Alexander biquandles. Then for any $x_{0}, x_{1}, \ldots, x_{n} \in X$, the assignment of them to each semi-arc of $\left(D_{A_{n}}, \phi_{A_{n}}\right)$ as shown in Figure 15 is an $X$-coloring of $\left(D_{A_{n}}, \phi_{A_{n}}\right)$, which implies $\operatorname{dim} \operatorname{Col}_{X}\left(D_{A_{n}}, \phi_{A_{n}}\right) \geq n+1$. By Corollary 6.2 , we obtain $n \leq u\left(A_{n}\right)$. On the other hand, we can deform $A_{n}$ into a trivial handlebody-knot by the crossing changes at $n$ crossings surrounded by dotted circles depicted in Figure 15. Therefore it follows that $u\left(A_{n}\right)=n$.

2. Let $s=t^{2}+1 \in \mathbb{Z}_{5}\left[t^{ \pm 1}\right]$ and let $f(t)=t^{2}+2 t+4 \in \mathbb{Z}_{5}\left[t^{ \pm 1}\right]$, which is an irreducible polynomial. Then $X:=\mathbb{Z}_{5}\left[t^{ \pm 1}\right] /(f(t))$ is a $\mathbb{Z}_{24}$-family of Alexander biquandles. Then for any $x_{0}, x_{1}, \ldots, x_{n} \in X$, the assignment of them to each semi-arc of $\left(D_{B_{n}}, \phi_{B_{n}}\right)$ as shown in Figure 16 is an $X$-coloring of $\left(D_{B_{n}}, \phi_{B_{n}}\right)$, which implies $\operatorname{dim} \operatorname{Col}_{X}\left(D_{B_{n}}, \phi_{B_{n}}\right) \geq n+1$. By Corollary 6.2 , we obtain $n \leq u\left(B_{n}\right)$. On the other hand, we can deform $B_{n}$ into a trivial handlebody-knot by the crossing changes at $n$ crossings surrounded by dotted circles depicted in Figure 16. Therefore it follows that $u\left(B_{n}\right)=n$. 


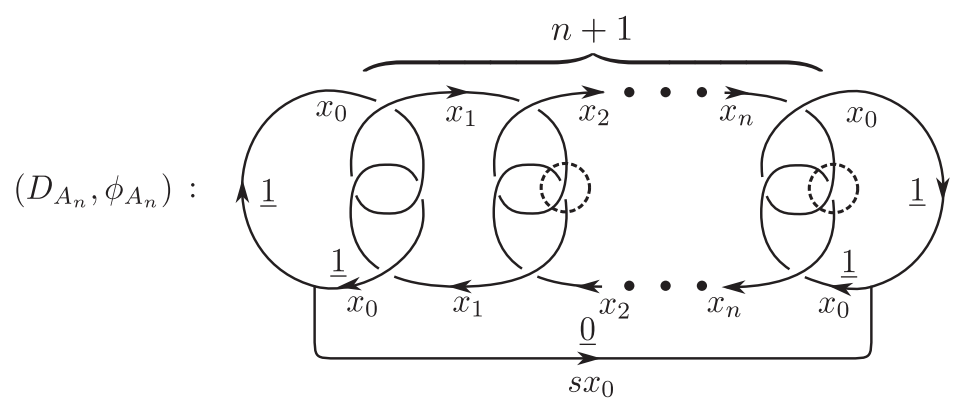

Figure 15. A $\mathbb{Z}_{8}$-flowed diagram $\left(D_{A_{n}}, \phi_{A_{n}}\right)$ of $A_{n}$.

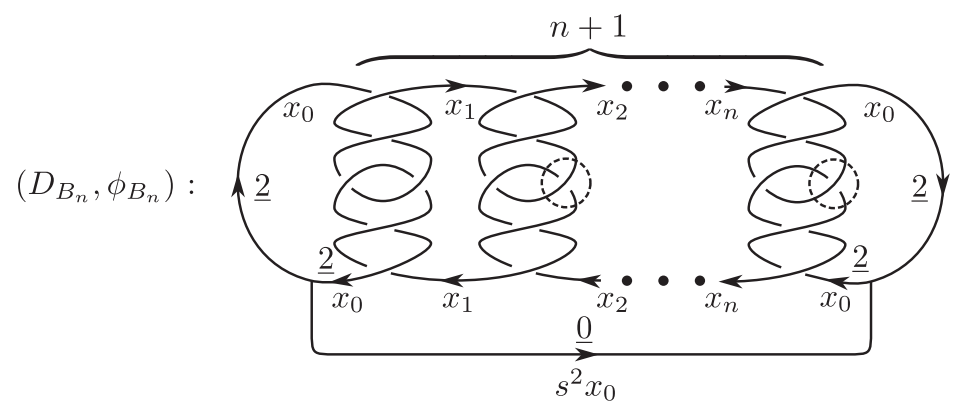

Figure 16. A $\mathbb{Z}_{24}$-flowed diagram $\left(D_{B_{n}}, \phi_{B_{n}}\right)$ of $B_{n}$.

3. Let $s=2 t-1 \in \mathbb{Z}_{3}\left[t^{ \pm 1}\right]$ and let $f(t)=t^{2}+t+2 \in \mathbb{Z}_{3}\left[t^{ \pm 1}\right]$, which is an irreducible polynomial. Then $X:=\mathbb{Z}_{3}\left[t^{ \pm 1}\right] /(f(t))$ is a $\mathbb{Z}_{8}$-family of Alexander biquandles. Then for any $x_{0}, x_{1}, \ldots, x_{n} \in X$, the assignment of them to each semi-arc of $\left(D_{C_{n}}, \phi_{C_{n}}\right)$ as shown in Figure 17 is an $X$-coloring of $\left(D_{C_{n}}, \phi_{C_{n}}\right)$, which implies $\operatorname{dim} \operatorname{Col}_{X}\left(D_{C_{n}}, \phi_{C_{n}}\right) \geq n+1$. By Corollary 6.2 , we obtain $n \leq u\left(C_{n}\right)$. On the other hand, we can deform $C_{n}$ into a trivial handlebody-knot by the crossing changes at $n$ crossings surrounded by dotted circles depicted in Figure 17. Therefore it follows that $u\left(C_{n}\right)=n$.

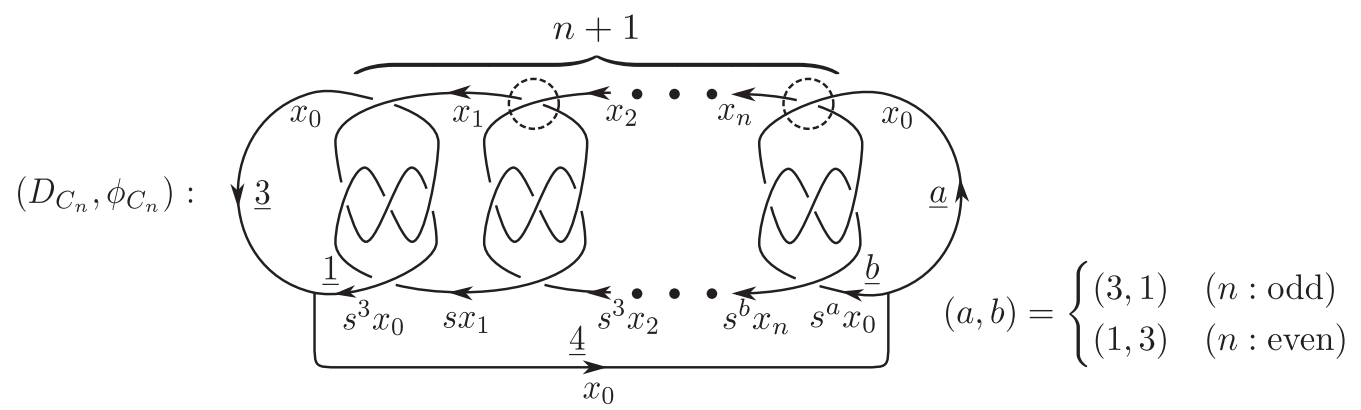

Figure 17. A $\mathbb{Z}_{8}$-flowed diagram $\left(D_{C_{n}}, \phi_{C_{n}}\right)$ of $C_{n}$.

EXAMPLE 7.4. Let $H_{n}$ and $H_{n}^{\prime}$ be the handlebody-knots represented by the $\mathbb{Z}_{3^{-}}$ 
flowed diagrams $\left(D_{n}, \phi_{n}\right)$ and $\left(D_{n}^{\prime}, \phi_{n}^{\prime}(a, b)\right)$ respectively depicted in Figure 18 for any $n \in \mathbb{Z}_{>0}$ and $a, b \in \mathbb{Z}_{3}$. Then we show that $d\left(H_{n}, H_{n}^{\prime}\right)=n$.

Let $s=1 \in \mathbb{Z}_{2}\left[t^{ \pm 1}\right]$ and let $f(t)=t^{2}+t+1 \in \mathbb{Z}_{2}\left[t^{ \pm 1}\right]$, which is an irreducible polynomial. Then $X:=\mathbb{Z}_{2}\left[t^{ \pm 1}\right] /(f(t))$ is a $\mathbb{Z}_{3}$-family of Alexander (bi)quandles. Then for any $x_{0}, x_{1}, \ldots, x_{n}, y_{1}, \ldots, y_{n} \in X$, the assignment of them to each semi-arc of $\left(D_{n}, \phi_{n}\right)$ as shown in Figure 18 is an $X$-coloring of $\left(D_{n}, \phi_{n}\right)$, which implies $\operatorname{dim} \operatorname{Col}_{X}\left(D_{n}, \phi_{n}\right) \geq$ $2 n+1$.

On the other hand, we note that $\operatorname{Col}_{X}\left(D_{n}^{\prime}, \phi_{n}^{\prime}(a, b)\right)$ is generated by $x_{0}, x_{1}, x_{1}^{\prime}, \ldots, x_{n}, x_{n}^{\prime}, y_{1}, y_{1}^{\prime}, \ldots, y_{n}, y_{n}^{\prime} \in X$ as shown in Figure 18 for any $a, b \in \mathbb{Z}_{3}$. If $(a, b)=(0,0)$, it is easy to see that $\operatorname{dim}^{C^{\prime}} \operatorname{Col}_{X}\left(D_{n}^{\prime}, \phi_{n}^{\prime}(a, b)\right)=1$. If $(a, b)=$ $(1,1),(1,2),(2,1),(2,2)$, we obtain that $x_{i}=x_{i}^{\prime}=y_{i}=y_{i}^{\prime}$ for any $i=1,2, \ldots, n$, which implies $\operatorname{dim} \operatorname{Col}_{X}\left(D_{n}^{\prime}, \phi_{n}^{\prime}(a, b)\right) \leq n+1$. If $(a, b)=(0,1),(0,2)$, we have

$$
\begin{aligned}
& x_{0}=x_{1}=x_{2}, \\
& x_{i+2}=x_{i}^{\prime}(i=1,2, \ldots, n-2) \text {, } \\
& x_{i}^{\prime}=\left\{\begin{array}{l}
x_{i} \underline{*}^{b} y_{i}^{\prime}(i: \text { odd }), \\
x_{i} \underline{*}^{-b} y_{i}^{\prime}(i: \text { even }),
\end{array}\right. \\
& x_{n}=x_{n-1}^{\prime} \text {, } \\
& y_{i}=y_{i}^{\prime}(i=1,2, \ldots, n) .
\end{aligned}
$$
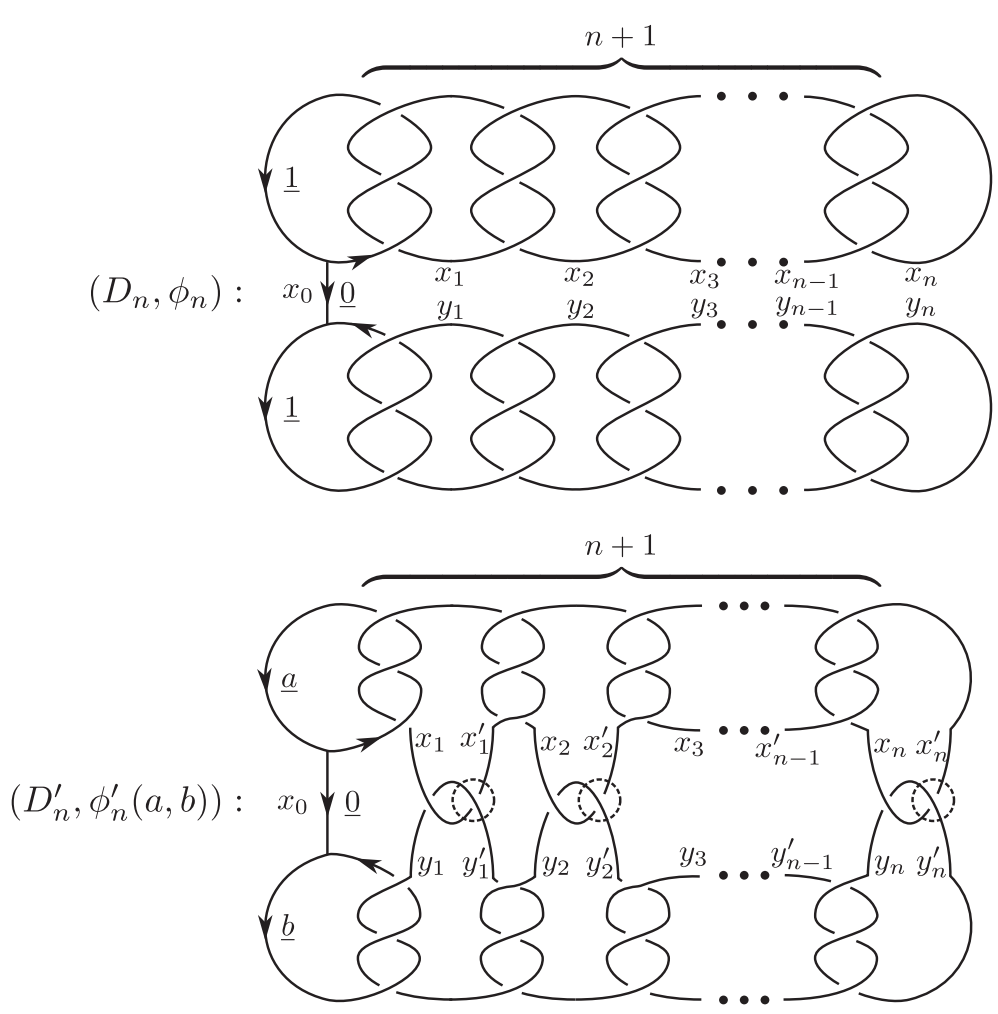

Figure 18. $\mathbb{Z}_{3}$-flowed diagrams $\left(D_{n}, \phi_{n}\right)$ and $\left(D_{n}^{\prime}, \phi_{n}^{\prime}\right)$ of $H_{n}$ and $H_{n}^{\prime}$. 
Hence $\operatorname{Col}_{X}\left(D_{n}^{\prime}, \phi_{n}^{\prime}(a, b)\right)$ is generated by $x_{0}, y_{1}, \ldots, y_{n} \in X$, which implies $\operatorname{dim} \operatorname{Col}_{X}\left(D_{n}^{\prime}, \phi_{n}^{\prime}(a, b)\right) \leq n+1$. If $(a, b)=(1,0),(2,0)$, in the same way as when $(a, b)=(0,1),(0,2), \operatorname{Col}_{X}\left(D_{n}^{\prime}, \phi_{n}^{\prime}(a, b)\right)$ is generated by $x_{0}, x_{1}, \ldots, x_{n} \in X$, which implies $\operatorname{dim} \operatorname{Col}_{X}\left(D_{n}^{\prime}, \phi_{n}^{\prime}(a, b)\right) \leq n+1$. Hence for any $a, b \in \mathbb{Z}_{3}, \operatorname{dim}_{\operatorname{Col}_{X}}\left(D_{n}^{\prime}, \phi_{n}^{\prime}(a, b)\right) \leq n+1$, which implies that

$$
\operatorname{dim} \operatorname{Col}_{X}\left(D_{n}, \phi_{n}\right)-\operatorname{dim} \operatorname{Col}_{X}\left(D_{n}^{\prime}, \phi_{n}^{\prime}(a, b)\right) \geq n .
$$

By Theorem 6.1, it follows that $n \leq d\left(H_{n}, H_{n}^{\prime}\right)$.

Finally, we can deform $H_{n}^{\prime}$ into $H_{n}$ by the crossing changes at $n$ crossings surrounded by dotted circles depicted in Figure 18. Therefore it follows that $d\left(H_{n}, H_{n}^{\prime}\right)=n$.

Acknowledgements. The author would like to thank Masahide Iwakiri for his helpful comments. He is particularly grateful to Atsushi Ishii for invaluable advice and his suggestions.

\section{References}

[1] J. W. Alexander, A lemma on systems of knotted curves, Proc. Nat. Acad. Sci. USA, 9 (1923), 93-95.

[ 2 ] W. E. Clark, M. Elhamdadi, M. Saito and T. Yeatman, Quandle colorings of knots and applications, J. Knot Theory Ramifications, 23 (2014), 1450035, 29 pp.

[ 3 ] R. Fenn, C. Rourke and B. Sanderson, Trunks and classifying spaces, Appl. Categ. Structures, 3 (1995), 321-356.

[4] A. Ishii, Moves and invariants for knotted handlebodies, Algebr. Geom. Topol., 8 (2008), 14031418.

[5] A. Ishii, The Markov theorems for spatial graphs and handlebody-knots with Y-orientations, Internat. J. Math., 26 (2015), 1550116, 23 pp.

[6] A. Ishii and M. Iwakiri, Quandle cocycle invariants for spatial graphs and knotted handlebodies, Canad. J. Math., 64 (2012), 102-122.

[ 7 ] A. Ishii, M. Iwakiri, Y. Jang and K. Oshiro, A $G$-family of quandles and handlebody-knots, Illinois J. Math., 57 (2013), 817-838.

[8] A. Ishii, M. Iwakiri, S. Kamada, J. Kim, S. Matsuzaki and K. Oshiro, A multiple conjugation biquandle and handlebody-links, Hiroshima Math. J., 48 (2018), 89-117.

[ 9 ] A. Ishii and K. Kishimoto, The IH-complex of spatial trivalent graphs, Tokyo. J. Math., 33 (2010), $523-535$.

[10] A. Ishii, K. Kishimoto, H. Moriuchi and M. Suzuki, A table of genus two handlebody-knots up to six crossings, J. Knot Theory Ramifications, 21 (2012), 1250035, 9 pp.

[11] A. Ishii and S. Nelson, Partially multiplicative biquandles and handlebody-knots, Contemp. Math., 689 (2017), 159-176.

[12] M. Iwakiri, Unknotting numbers for handlebody-knots and Alexander quandle colorings, J. Knot Theory Ramifications, 24 (2015), 1550059, 13 pp.

[13] D. Joyce, A classifying invariant of knots, the knot quandle, J. Pure Appl. Alg., 23 (1982), 37-65.

[14] S. V. Matveev, Distributive groupoids in knot theory, Mat. Sb. (N.S.), 119(161) (1982), 78-88.

[15] T. Murao, On bind maps for braids, J. Knot Theory Ramifications, 25 (2016), 1650004, 25 pp.

[16] Y. Nakanishi, A note on unknotting number, Math. Sem. Notes Kobe Univ., 9 (1981), 99-108.

[17] J. Przytycki, 3-coloring and other elementary invariants of knots, Banach Center Publ., 42 (1998), 275-295. 


\section{Tomo Murao}

Institute of Mathematics

University of Tsukuba

1-1-1 Tennodai, Tsukuba

Ibaraki 305-8571, Japan

E-mail: t-murao@math.tsukuba.ac.jp 